\title{
THE HAUPTVERMUTUNG AND THE POLYHEDRAL SCHOENFLIES THEOREM
}

\author{
BY P. M. RICE
}

\author{
Communicated by M. L. Curtis, December 28, 1964
}

1. Introduction. M. L. Curtis [1] has conjectured that the double suspension of a Poincaré manifold is a 5 -sphere. If this is true, it gives counterexamples to the Hauptvermutung, the closed star conjecture, and the polyhedral Schoenflies theorems. We prove here that the only way to get a noncombinatorial triangulation of a manifold is, essentially, to multiply suspend a combinatorial manifold which is not a sphere. As a corollary, we establish that, modulo the Poincaré conjecture, one of the polyhedral Schoenflies theorems is equivalent to the Hauptvermutung.

2. Terminology. The Hauptvermutung is the conjecture that any two triangulations of an $n$-manifold are piecewise linearly homeomorphic. It is convenient to consider two conjectures which together imply the Hauptvermutung. The first is that any triangulation of an $n$-manifold is combinatorial (meaning that the link of any vertex is a combinatorial (n-1)-sphere), and the second is that any two combinatorial triangulations of an $n$-manifold are piecewise linearly homeomorphic. We will call the first of these $H(n) . H(n)$ is known for $n=1,2,3$. PS $(n)$ will denote the conjecture that, if a combinatorial $(n-1)$-sphere $S$ is embedded as a subcomplex of a triangulated $n$ sphere $T$, then $S$ is locally flat in $T$. PS $(n)$ is known for $n=1,2,3$. $P(n)$ will be the $n$-dimensional Poincaré conjecture, which is known except for $n=3,4$. $S^{n}$ will be any space homeomorphic to the $n$-sphere, $X \cong Y$ means $X$ is homeomorphic to $Y, X \circ Y$ is the topological join of $X$ and $Y$, and $S(X)$ is the suspension of $X$.

\section{Main result.}

THEOREM. If there is a noncombinatorial triangulation of an n-manifold $M$, then there is a combinatorial $m$-manifold $K^{m}, m \geqq 3$, such that

(i) $K^{m}$ is a homology $m$-sphere but $K^{m} \neq S^{m}$ and

(ii) $K^{m} \circ S^{n-m-1} \cong S^{n}$.

Proof. Let $v$ be a vertex of $M$ such that $\operatorname{LK}(v, M)$, the link of $v$ in $M$, is not a combinatorial $(n-1)$-sphere. If $\operatorname{LK}(v, M)=K^{n-1}$ is a combinatorial manifold, then $S\left(K^{n-1}\right) \cong S^{n}$ by Theorem 4 of [2] and the theorem is proved. By induction, if $K^{p} \circ S^{n-p-1} \cong S^{n}$ but $K^{p}$ is not 
a combinatorial manifold, then, for some vertex $w$ of $K^{p}, \mathrm{LK}\left(w, K^{p}\right)$ $=K^{p-1}$ is not a combinatorial $(p-1)$-sphere. $\operatorname{But} \operatorname{St}\left(w, K^{p}\right)=w \circ K^{p-1}$ and $\operatorname{St}\left(w, K^{p}\right) \circ S^{n-p-1} \cong E^{n}$, so $\left(w \circ K^{p-1}\right) \circ S^{n-p-1}=w \circ\left(K^{p-1} \circ S^{n-p-1}\right)$ is locally $n$-euclidean at $w$, and, by Theorem 4 of [2],

$$
S\left(K^{p-1} \circ S^{n-p-1}\right)=K^{p-1} \circ S^{n-p} \cong S^{n} .
$$

For some $m \geqq 3, K^{m}$ is a combinatorial manifold, for, if not, then $K^{2} \circ S^{n-3} \cong S^{n}$, so $K^{2}$ is a polyhedral homology manifold with the homology of $S^{2}$, whence $K^{2} \cong S^{2}$, and, since $H(2)$ is true, $K^{2}$ is a combinatorial 2-sphere, from which it would follow that $K^{3}$ is a combinatorial manifold.

Corollary 1. Suppose that a combinatorial (n-1)-sphere is embedded as a subcomplex of a triangulated $n$-sphere such that the closure of one complementary domain is a combinatorial n-cell. If this implies that the other complementary domain is simply connected, then $P(3)$ and $P(4)$ imply $H(n)$.

PROoF. Let $K^{m}$ be as in the theorem, and $v$ a vertex of $K^{m}$. St $\left(v, K^{m}\right)$ $\circ S^{n-m-1}$ is a combinatorial $n$-cell embedded as a subcomplex of $K^{m} \circ S^{n-m-1} \cong S^{n}$. Then

$$
\left(K^{m} \circ S^{n-m-1}\right) \backslash\left(\operatorname{St}\left(v, K^{m}\right) \circ S^{n-m-1}\right)=\left(K^{m} \backslash \operatorname{St}\left(v, K^{m}\right)\right) \times E^{n-m}
$$

is simply connected. Thus $K^{m}$ is a simply connected, combinatorial, homology sphere, which, by the Poincaré conjecture, is an $m$-sphere.

Corollary 2. $P(3), P(4), P S(n)$ implies $H(n)$, and $H(n)$ implies $P S(n)$.

PRoof. The last statement follows from the combinatorial Schoenflies theorem.

\section{REFERENCES}

1. M. L. Curtis and E. C. Zeeman, On the polyhedral Schoenflies theorem, Proc. Amer. Math. Soc. 11 (1960), 888-889.

2. R. H. Rosen, Stellar neighborhoods in polyhedral manifolds, Proc. Amer. Math. Soc. 14 (1963), 401-406.

UNIVERSITY OF GEORGIA 